Pacific Journal of Mathematic 


\title{
SOME SPECIAL EQUATIONS IN A FINITE FIELD
}

\author{
L. CA R LIT Z
}

1. The equation (1.1). Let $f_{i}(u), i=1, \cdots, r$ denote polynomials with coefficients in the finite field $G F(q), q=p^{n}$. We consider the equation

$$
f_{1}\left(\xi_{1}\right)+\cdots+f_{r}\left(\xi_{r}\right)=\alpha \quad\left(\xi_{i}, \alpha \in G F(q)\right)
$$

let $N$ denote the number of solutions of (1.1).

For $\beta \in G F(q)$, put

$$
e(\beta)=e^{2 \pi i t(\beta) / p}, \quad t(\beta)=\beta+\beta^{p}+\cdots+\beta^{p^{n-1}} .
$$

Then we may write

$$
q N=\sum_{\beta} e(-\alpha \beta) \sum_{\xi_{1}, \cdots, \xi_{r}} e\left(\beta f_{1}\left(\xi_{1}\right)+\cdots+\beta f_{r}\left(\xi_{r}\right)\right),
$$

where the summation extends over all numbers $\beta, \xi_{i}$ of $G F(q)$. Now put

$$
S(f)=\sum_{\xi} e(f(\xi))
$$

where $f$ is any polynomial with coefficients in $G F(q)$. Then (1.2) becomes

$$
q N=q^{r}+\sum_{\beta \neq 0} e(-\alpha \beta) \prod_{i=1}^{r} S\left(\beta f_{i}\right)
$$

2. Estimate for $N$. If $\operatorname{deg} f \leq 2, S(f)$ can be evaluated explicitly. However, we are primarily interested in the case $\operatorname{deg} f>2$. An estimate for $S(f)$ is given by the following:

Тнеовем 1. If $k=\operatorname{deg} f<p$, then

$$
S(f)=O\left(q^{1-1 / k}\right)
$$

Received March 19, 1952.

Pacific J. Math. 3 (1953), 13- 24 
Mordell [7] has proved (2.1) in the case $n=1$, that is, $q=p$. However, examination of his proof shows that $(2.1)$ holds for all $n \geq 1$ provided we have $\operatorname{deg} f<p$.

If we substitute from $(2.1)$ in (1.4) we have at once:

THEOREM 2. The number of solutions of (1.1), where $\operatorname{deg} f_{i}=k_{i}<p$, is given by

$$
N=q^{r-1}+O\left(q^{r-w}\right)
$$

$$
\left(w=\frac{1}{k_{1}}+\cdots+\frac{1}{k_{r}}\right) .
$$

This result is trivial unless $w>1$, which will evidently be satisfied for $r$ sufficiently large.

Hua and Vandiver [5] and Weil [9] have discussed the number of solutions of $(1.1)$ in the special case $f_{i}(x)=x^{k_{i}}$; their results are considerably better than $(2.2)$.

If $g_{i}(u), i=1, \cdots, r$, denote a second set of polynomials with coefficients in $G F(q)$ and such that $\operatorname{deg} g_{i}<k_{i}$, then an estimate can be obtained for the weighted sum

$$
S_{g}=\sum_{\xi_{1}, \cdots, \xi_{r}} e\left(g_{1}\left(\xi_{1}\right)+\cdots+g_{r}\left(\xi_{r}\right)\right),
$$

where the summation is extended over all $\xi_{i}$ satisfying (1.1). Indeed, we have

$$
\begin{aligned}
q S_{g} & =\sum_{\beta} e(-\alpha \beta) \sum_{\xi_{1}}, \ldots, \xi_{r} e\left\{\sum_{i=1}^{r}\left(\beta f_{i}\left(\xi_{i}\right)+g_{i}\left(\xi_{i}\right)\right)\right\} \\
& =\sum_{\beta} e(-\alpha \beta) \prod_{i=1}^{r} S\left(\beta f_{i}+g_{i}\right),
\end{aligned}
$$

in the notation of (1.3); consequently if at least one $g_{i}(x)$ is of degree $\geq 1$, it follows that

$$
S_{g}=O\left(q^{r-w}\right)
$$

$$
\left(w=\frac{1}{k_{1}}+\cdots+\frac{1}{k_{r}}\right) .
$$

If all $k_{i}=2$ then an explicit formula can be obtained for $S_{g}$. 


$$
f(x)=\alpha_{1} x^{e_{1}}+\cdots+\alpha_{k} x^{e_{k}} \quad\left(\alpha_{i} \in G F(q)\right)
$$

Mordell has proved that

$$
S(f)=O\left(q^{1-1 /(2 k)}\right) \quad(q \rightarrow \infty)
$$

in the special case $q=p$. Negative values of $e_{i}$ are permitted; however, in that case it is assumed that in the definition of $S(f)$, the summation is over $\xi \neq 0$. Clearly this does not affect the estimate (3.2). Here again we find that Mordell's proof applies to the general case. We state:

THEOREM 3. If the integers $e_{i}$ in $(3.1)$ are incongruent $(\bmod q-1)$, then (3.2) holds.

(We remark that Min $[6, p .139$, Lemma 1$]$ states that $(2.1)$ is valid, without mentioning the restriction $k<p$. However, his proof does not seem adequate. For example, for $k=p$, the system

$$
\sum_{i=1}^{p} x_{i}^{j}=\sum_{i=1}^{p} y_{i}^{j}
$$

does not imply that the $y$ 's are a permutation of the $x$ 's.)

By means of Theorem 3 we obtain at once:

THEOREM 4. Let $f_{i}(x), i=1, \cdots, r$, be polynomials of the type (3.1), with $k$ replaced by $k_{i}$, and let no two exponents in $f_{i}(x)$ be congruent (mod $q-1)$. Then the number of solutions of

$$
f_{1}\left(\xi_{1}\right)+\cdots+f_{r}\left(\xi_{r}\right)=\alpha
$$

is given by

$$
q^{r-1}+O\left(q^{r-w}\right)
$$

$$
\left(w=\frac{1}{2 k_{1}}+\cdots+\frac{1}{2 k_{r}}\right) .
$$

Once again we have $w>1$ for $r$ sufficiently large.

The most interesting case of (3.1) is perhaps $f(x)=\alpha x+\beta x^{-1}$. The corresponding sum $S(f)$ is the Kloosterman sum

$$
K(\alpha, \beta)=\sum_{\xi \neq 0} e\left(\alpha \xi+\beta \xi^{-1}\right) .
$$


Theorem 4 now implies:

THEOREM 5. The number of solutions $\xi_{i} \neq 0$ of

$$
\alpha_{1} \xi_{1}+\frac{\beta_{1}}{\xi_{1}}+\cdots+\alpha_{r} \xi_{r}+\frac{\beta_{r}}{\xi_{r}}=\alpha
$$

is given by

$$
q^{r-1}+O\left(q^{3 r / 4}\right)
$$

Indeed if we make use of Andre' Weil's estimate [10] for (3.5)

$$
|K(\alpha, \beta)| \leq 2 q^{1 / 2}
$$

then (3.7) can be replaced by

$$
q^{r-1}+O\left(q^{r / 2}\right)
$$

which is significant for $r \geq 3$.

4. Another special case. Let $p>2$. Theorem 4 applies to $f(x)=x^{2}+x^{-2}$, and indeed ( 3.7 ) furnishes on asymptotic formula for the number of solutions of

$$
\alpha_{1} \xi_{1}^{2}+\frac{\beta_{1}}{\xi_{1}^{2}}+\cdots+\alpha_{r} \xi_{r}^{2}+\frac{\beta_{r}}{\xi_{r}^{2}}=\alpha \quad\left(\alpha \alpha_{i} \beta_{i} \neq 0\right)
$$

However it is of interest to note that certain exact results can be obtained. Let $N_{1}$ and $N_{2}$ denote the number of solutions of (3.6) and (4.1), respectively. On the one hand

$$
q N_{1}=q^{r}+\sum_{\beta \neq 0} e(-\alpha \beta) \prod_{i=1}^{r} K\left(\beta \alpha_{i}, \beta \beta_{i}\right)
$$

on the other hand

$$
q N_{2}=q^{r}+\sum_{\beta \neq 0} e(-\alpha \beta) \prod_{i=1}^{r} K_{2}\left(\beta \alpha_{i}, \beta \beta_{i}\right)
$$

where 


$$
K_{2}(\alpha, \beta)=\sum_{\xi \neq 0} e\left(\alpha \xi^{2}+\beta \xi^{-2}\right)
$$

Let $\psi(\xi)=+1$ or -1 according as $\xi$ is a square or a non-square of $G F(q)$. Then (4.4) implies

$$
K_{2}(\alpha, \beta)=\sum_{\xi \neq 0}(1+\psi(\xi)) e\left(\alpha \xi+\beta \xi^{-1}\right)=K(\alpha, \beta)+L(\alpha, \beta),
$$

where

$$
L(\alpha, \beta)=\sum_{\xi \neq 0} \psi(\xi) e\left(\alpha \xi+\beta \xi^{-1}\right) .
$$

Now it is not difficult to evaluate $L(\alpha, \beta)$ explicitly (compare [8, p.102]). We have

$$
L(\alpha, \beta)=\left\{\begin{array}{lc}
0 & (\psi(\alpha \beta)=-1) \\
G(1)(e(2 \gamma)+e(-2 \gamma)) & \left(\alpha \beta=\gamma^{2}\right) .
\end{array}\right.
$$

As for the Gauss sum $G(1)$, we note $[1, \S 3]$

(4.7) $G(\alpha)=\sum_{\xi} e\left(\alpha \xi^{2}\right)=\psi(\alpha) G(1) \quad\left(\alpha \neq 0 ; G^{2}(1)=q \psi(-1)\right)$.

Then by (4.3), (4.4), and (4.5),

(4.8) $\quad q N_{2}=q^{r}+\sum_{\beta \neq 0} e(-\alpha \beta) \prod_{i=1}^{r}\left(k\left(\beta \alpha_{i}, \beta \beta_{i}\right)+L\left(\beta \alpha_{i}, \beta \beta_{i}\right)\right)$.

Comparison of (4.2) and (4.8) leads at once to:

THEOREM 6. If $\psi\left(\alpha_{i} \beta_{i}\right)=-1, i=1, \cdots, r$, then the number of solutions of (4.1) is equal to the number of solutions of (3.6).

5. Quadratic forms. In the remainder of the paper we shall be concerned with a quadratic form

$$
Q\left(u_{1}, \ldots, u_{r}\right)=\sum_{i, j=1}^{r} \alpha_{i j} u_{i} u_{j} \quad\left(\alpha_{i j} \in G F(q), \delta=\left|\alpha_{i j}\right| \neq 0\right) .
$$

We recall that the number of solutions $N_{Q}(\alpha)$ of 


$$
Q\left(\xi_{1}, \cdots, \xi_{r}\right)=\alpha
$$

is given $[4, \mathrm{pp} .47-48]$ by

$$
\left\{\begin{array}{l}
q^{2 s-1}+\left(q^{s}-q^{s-1}\right) \psi\left((-1)^{s} \delta\right) \\
q^{2 s-1}-q^{s-1} \psi\left((-1)^{s} \delta\right)
\end{array}\right.
$$

for $r=2 s$;

$$
q^{2 s}+q^{s} \psi\left((-1)^{s} \alpha \delta\right)
$$

for $r=2 s+1$, where in $(5.4)$ it is understood that $\psi(0)=0$.

Now let $f\left(u_{1}, \cdots, u_{t}\right)$ denote an arbitrary polynomial with coefficients in $G F(q)$, and let $N_{f}(\alpha)$ denote the number of solutions $\zeta_{1}$ of

$$
f\left(\zeta_{1}, \cdots, \zeta_{t}\right)=\alpha
$$

Clearly the number of solutions $\xi_{i}, \zeta_{j}$ of

$$
Q\left(\xi_{1}, \ldots, \xi_{t}\right)=f\left(\zeta_{1}, \ldots, \zeta_{t}\right)
$$

is given by

$$
N=\sum_{a} N_{Q}(\alpha) N_{f}(\alpha)
$$

We shall now show that the right member of (5.7) can be evaluated in certain cases.

In the first place let $f=u^{k}$. Then $(5.7)$ becomes

$$
N=\sum_{\xi} N_{Q}\left(\xi^{k}\right)=N_{Q}(0)+\sum_{\xi \neq 0} N_{Q}\left(\xi^{k}\right) .
$$

Now apply (5.3) and we get, for $r=2 s$,

$$
N=\left(q^{2 s-1}+\left(q^{s}-q^{s-1}\right) \psi\left((-1)^{s} \delta\right)\right)+(q-1)\left(q^{2 s-1}-q^{s-1} \psi\left((-1)^{s} \delta\right)\right)
$$

which is simply

$$
N=q^{2 s}
$$


Similarly, application of (5.4) in the case $r=2 s+1$ yields

$$
N=\left\{\begin{array}{l}
q^{2 s+1} \\
q^{2 s+1}+q^{s}(q-1) \psi\left((-1)^{s} \delta\right)
\end{array}\right.
$$

This proves:

THEOREM 7. The number of solutions of

$$
Q\left(\xi_{1}, \ldots, \xi_{r}\right)=\zeta^{k}
$$

is furnished by (5.8) and (5.9).

A slight generalization of Theorem 7 is contained in:

THEOREM 8. The number of solutions of

$$
Q\left(\xi_{1}, \ldots, \xi_{r}\right)=\zeta_{1}^{k_{1}} \cdots \zeta_{t}^{k_{t}}
$$

is given by

$$
N=q^{t+2 s-1}+\left\{\left(q^{s}-q^{s-1}\right) q^{t}-q^{s}(q-1)^{t}\right\} \psi\left((-1)^{s} \delta\right)
$$

for $r=2 s$

$$
N=\left\{\begin{array}{lc}
q^{t+2 s} & \left(\left(k_{1}, \ldots, k_{t}\right) \text { odd }\right) \\
q^{t+2 s}+q^{s}(q-1)^{t} \psi\left((-1)^{s} \delta\right) & \left(\left(k_{1}, \ldots, k_{t}\right) \text { even }\right)
\end{array}\right.
$$

for $r=2 s+1$.

In the next place let $f$ denote a polynomial such that $f\left(\zeta_{2}, \cdots, \zeta_{t}\right)$ never vanishes. Then since for $r=2 s, \alpha \neq 0, N_{Q}(\alpha)$ is independent of $\alpha$, we see that the number of solutions of (5.6) is given by

$$
N=q^{t}\left\{q^{2 s-1}-q^{s-2} \psi\left((-1)^{s} \delta\right)\right\}
$$

for $r=2 s$. On the other hand, for $r=2 s+1$ we get

$$
N=q^{t+3 s}+q^{s} \psi\left((-1)^{s} \delta\right) \sum_{\zeta_{1}}, \ldots, \zeta_{t} \psi\left(f\left(\zeta_{1}, \ldots, \zeta_{t}\right)\right)
$$


We state:

THEOREM 9. Let $f$ be a polynomial such that $f\left(\zeta_{1}, \cdots, \zeta_{t}\right)$ never vanishes. Then the number of solutions of (5.6) is furnished by (5.10) and (5.11).

Note that the right member of $(5.10)$ is independent of the polynomial $f$. It follows from (5.11) that the number of solutions of

$$
Q\left(\xi_{1}, \ldots, \xi_{2 s+1}\right)=f^{2 m+1}\left(\zeta_{1}, \ldots, \zeta_{t}\right)
$$

is the same for all values of $m$. Other special cases that lead to simple explicit results are contained in the following two theorems:

THEOREM 10. Let $f$ be a polynomial such that $f\left(\zeta_{1}, \cdots, \zeta_{t}\right)$ never vanishes. Then the number of solutions of

$$
Q\left(\xi_{1}, \ldots, \xi_{2 s+1}\right)=f^{2}\left(\zeta_{1}, \ldots, \zeta_{t}\right)
$$

is given by

$$
q^{t+2 s}+q^{t+s} \psi\left((-1)^{s} \delta\right)
$$

THEOREM 11. Let $f$ be a polynomial such that $f\left(\zeta_{1}, \ldots, \zeta_{t}\right)$ never vanishes. Then the number of solutions $\xi_{i}, \eta, \zeta_{j}$ of

$$
Q\left(\xi_{1}, \cdots, \xi_{r}\right)=\eta^{k} f\left(\zeta_{1}, \ldots, \zeta_{t}\right)
$$

is $q^{t+2 s}$ for $r=2 s$, while for $r=2 s+1$ the number of solutions is given by

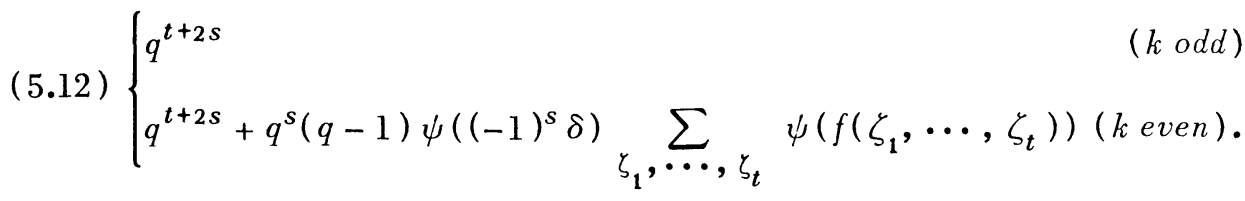

In particular if $f$ is the square of a polynomial then the second of (5.12) reduces to

$$
q^{t+2 s}+q^{t+s}(q-1) \psi\left((-1)^{s} \delta\right)
$$

It is clear how Theorem 11 can be generalized to give the number of solutions of 


$$
Q\left(\xi_{1}, \ldots, \xi_{r}\right)=\eta_{1}^{k_{1}} \ldots \eta_{w}^{k_{w}} f\left(\zeta_{1}, \ldots, \zeta_{t}\right)
$$

A word may be added about a generalization of a different kind. Let $Q_{i}$ denote quadratic forms in $r_{i}$ indeterminates and of discriminant $\delta_{i} \neq 0$. Then we can treat such equations as

$$
Q_{1}\left(\xi_{1}, \ldots, \xi_{r_{1}}\right) Q_{2}\left(\eta_{1}, \ldots, \eta_{r_{2}}\right)=\alpha
$$

For example, the number of solutions of (5.13) for $\alpha \neq 0$ is evidently

$$
\sum_{\beta \neq 0} N_{Q_{1}}(\beta) N_{Q_{2}}(\alpha / \beta),
$$

which can be evaluated by means of (5.3) and (5.4). In particular if $r_{1}$ and $r_{2}$ are both even, then $(5.14)$ becomes

$$
(q-1)\left(q^{2 s_{1}-1}-q^{s_{1}-1} \psi\left((-1)^{s_{1}} \delta_{1}\right)\right)\left(q^{2 s_{2}-1}-q^{s_{2}-1} \psi\left((-1)^{s_{2}} \delta_{2}\right)\right),
$$

where $r_{i}=2 s_{i}$. In similar fashion we can determine the number of solutions of, say,

$$
Q_{1} Q_{2}+\cdots+Q_{2 w-1} Q_{2 w}=\alpha
$$

where no two Q's have any unknowns in common.

6. Bounds $(t=1)$. Returning to (5.11) and (5.12), we remark that since an exact formula for such sums as

$$
\sum_{\zeta_{1}, \cdots, \zeta_{t}} \psi\left(f\left(\zeta_{1}, \cdots, \zeta_{t}\right)\right)
$$

is usually not available, it is natural to look for a bound. We shall consider only the case $t=1$. Then for the sum

$$
T(f)=\sum_{\zeta} f(\zeta)
$$

it follows from a theorem of Weil [10] that

$$
T(f)=O\left(q^{1 / 2}\right)
$$

by more elementary methods one can prove the weaker estimate [3] 


$$
T(f)=O\left(q^{1-\Theta_{k}}\right)
$$

where $\Theta_{3}=1 / 4, \Theta_{k}=3 / 2(k+4)$ for $k \geq 4$.

Thus applying (6.2) or (6.3) we obtain asymptotic results for (5.11) and (5.12) with $t=1$.

7. Extension of results of $\S 5$. The results of $\xi 5$ can be extended by making use of known results on the number of solutions of

$$
Q\left(U_{1}, \ldots, U_{r}\right)=\alpha
$$

in polynomials $U_{i} \in G F[q, x]$ of degree $<m$; $Q$ has its usual meaning. For simplicity we limit our attention to the case $r=2 s$. Cohen [2, p.556, Cor. 3] has proved that the number of solutions of (7.1) with $r=2 s$ is

$$
\begin{cases}\left(q^{s}-\lambda\right) q^{(s-1)(2 m-1)} & (\alpha \neq 0) \\ \lambda^{m} q^{m s}+\left(q^{s}-\lambda\right) q^{(s-1)(2 m-1)} \sum_{z^{s}=0}^{m-1} \lambda^{z} q^{-z(s-2)} & (\alpha=0),\end{cases}
$$

where $\lambda=\psi\left((-1)^{s} \delta\right)$. Then we have:

THEOREM 12. The number of solutions of

$$
Q\left(U_{1}, \ldots, U_{2 s}\right)=\zeta_{1}^{k_{1}} \ldots \zeta_{t}^{k_{t}}
$$

in polynomials $U_{i}$ of degree $<m$ is

$$
\begin{gathered}
\left(q^{t}-(q-1)^{t}\right)\left\{\lambda^{m} q^{m s}+\left(q^{s}-\lambda\right) q^{(s-1)(2 m-1)} \sum_{z=0}^{m-1} \lambda^{z} q^{-z(s-2)}\right\} \\
+(q-1)^{t}\left(q^{s}-\lambda\right) q^{(s-1)(2 m-1)}
\end{gathered}
$$

where $\lambda=\psi\left((-1)^{s} \delta\right)$.

The proof is like that of Theorem 7 .

THEOREM 13. Let $f$ be a polynomial such that $f\left(\zeta_{1}, \ldots, \zeta_{t}\right)$ never vanishes. Then the number of solutions of

$$
Q\left(U_{1}, \ldots, U_{2 s}\right)=f\left(\zeta_{1}, \ldots, \zeta_{t}\right)
$$


in polynomials $U_{i}$ of degree $<m$ and $\zeta_{j} \in G F(q)$ is

$$
q^{t+(s-1)(2 k-1)}\left(q^{s}-\lambda\right) \text {. }
$$

THEOREM 14. Let $f$ be a polynomial such that $f\left(\zeta_{1}, \ldots, \zeta_{t}\right)$ never vanishes. Then the number of solutions of

$$
Q\left(U_{1}, \cdots, U_{2 s}\right)=\eta_{1}^{k_{1}} \ldots \eta_{t}^{k_{t}} f\left(\zeta_{1}, \ldots, \zeta_{w}\right) \quad\left(k_{i} \geq 1\right),
$$

with $\operatorname{deg} U_{i}<m$, is $q^{w}$ times the number of solutions of (7.3).

The proof of these theorems is immediate.

Finally we mention problems like (5.13) and (5.15) in which the unknowns are polynomials. Thus for example the number of solutions of

$$
Q_{1}\left(U_{1}, \ldots, U_{2 s_{i}}\right) U_{2}\left(V_{1}, \ldots, V_{2 s_{2}}\right)=f\left(\zeta_{1}, \ldots, \zeta_{t}\right)
$$

with $\operatorname{deg} U_{i}<m_{i}$, $\operatorname{deg} U_{2}<m_{2}$, where $f$ never vanishes, is equal to

$$
q^{t+\left(s_{1}-1\right)\left(2 m_{1}-1\right)+\left(s_{2}-1\right)\left(2 m_{2}-1\right)}\left(q^{s_{1}}-\lambda_{1}\right)\left(q^{s_{2}}-\lambda_{2}\right)(q-1),
$$

where $\lambda_{i}=\psi\left((-1)^{s_{i}} \delta_{i}\right)$, and $\delta_{i}$ is the discriminant of $Q_{i}$.

It may also be mentioned that in a problem like (7.3) we may restrict some of the $U_{i}$ to be primary of degree $m$; the final formula is similar to that obtained in Theorem 12. The same remark applies to the other theorems of this section.

\section{REFERENCES}

1. L. Carlitz, The singular series for sums of squares of polynomials, Duke Math. J. 14 (1947), $1105-1120$.

2. Eckford Cohen, Sums of an even number of squares in $G F\left[p^{n}, x\right]$ II, Duke Math. J. 14 (1947), 545 - 557.

3. H. Davenport, On character sums in finite fields, Acta Math. 71 (1939), 99-121.

4. L. E. Dickson, Linear groups, Leipzig, 1901.

5. L. K. Hua and H. S. Vandiver, Characters over certain types of rings with applications to the theory of equations in a finite field, Proc. Nat. Acad. Sci., U.S.A. 35 (1949), $94-99$.

6. S. H. Min, On systems of algebraic equations and certain multiple exponential sums, Quart. J. Math. Oxford Ser. 18 (1947), $133-142$. 
7. L. J. Mordell, On a sum analogous to a Gauss's sum, Quart. J. Math. Oxford Ser. 3 (1932), 161 - 167.

8. H. Salié, Über die Kloostermanschen Summen $S(u, v ; q)$, Math. Z. 34 (1932), $91-109$.

9. André Weil, Numbers of solutions of equations in finite fields, Bull. Amer. Math. Soc. 55 (1949), 497-508.

10. André Weil, On some exponential sums, Proc. Nat. Acad. Sci. U.S.A. 34 (1948), $204-207$.

DUKE UNIVERSITY 


\section{PACIFIC JOURNAL OF MATHEMATICS}

\section{EDITORS}

\author{
R. M. Foeinson \\ University of California \\ Berkeley 4, California \\ E. HEWITt \\ University of Washington \\ Seattle 5, Washington
}

R. P. DILWOR TH

California Institute of Technology

Pasadena 4, California

E. F. BECKENBACH

University of California

Los Angeles 24, California

\section{ASSOCIATE EDITORS}

$\begin{array}{llll}\text { H. BUSEMANN } & \text { P. R. HALMOS } & \text { BøRGE JESSEN } & \text { J. J. STOKER } \\ \text { HERBERT FFDERER } & \text { HEINZ HOPF } & \text { PAUL LÉVY } & \text { E. G. STR AUS } \\ \text { MARSHALL IIALI } & \text { R. D. JAMES } & \text { GEORGE PÓLYA } & \text { KỎSAKU YOSIDA }\end{array}$

\section{SPONSORS}

UNIVERSITY OF BRITISH COLUMBIA CAIIFORNIA INSTITUTE OF TECHNOLOGY UNIVERSITY OF CALIFORNIA, BERKELEY UNIVERSITY OF CAILIFORNIA, DAVIS UNIVERSITY OF CALIFORNIA, LOS ANGELES UNIVERSITY OF CALIFORNIA, SANTA BARBARA UNIVERSITY OF NEVADA OREGON STATE COLLEGE UNIVERSITY OF OREGON
UNIVERSITY OF SOUTHERN CALIFORNIA STANFORD RESEARCH INSTITUTE STANFORD UNIVERSITY WASHINGTON STATE COLLEGE UNIVERSITY OF WASHINGTON

AMERICAN MATHEMATICAL SOCIETY NATIONAL BUREAU OF STANDARDS, INSTITUTE FOR NUMERICAL ANALYSIS

$$
\begin{gathered}
\text { Vari-Type Composition by } \\
\text { Elaine Barth } \\
\text { Delores Wierman } \\
\text { With the cooperation of } \\
\text { E. F. Beckenbach } \\
\text { E. G. Straus }
\end{gathered}
$$

Printed in the United States of America by Edwards Brothers, Inc., Ann Arbor, Michigan 


\section{Pacific Journal of Mathematics}

\section{Vol. 3, No. 1 \\ March, 1953}

Herbert Busemann, Volume in terms of concurrent cross-sections ......... 1

L. Carlitz, Some special equations in a finite field ................. 13

Homer V. Craig and Billie Braden Townsend, On certain metric

extensors ....................................... 25

Philip J. Davis and Henry Pollak, Linear functionals and analytic

continuation problems ............................. 47

Jacob C. E. Dekker, The constructivity of maximal dual ideals in certain

Boolean algebras ................................. 73

Harley M. Flanders, The norm function of an algebraic field extension .... 103

Marshall Hall, Subgroups of free products . . . . . . . . . . . . . . . . . 115

Israel (Yitzchak) Nathan Herstein, Finite multiplicative subgroups in

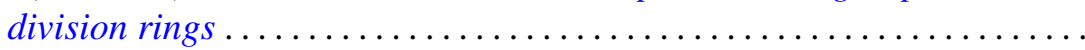

Joseph Lawson Hodges, Jr. and Murray Rosenblatt, Recurrence-time moments in random walks ............................ 127

Alfred Horn, The normal completion of a subset of a complete lattice and lattices of continuous functions ........................ 137

Fulton Koehler, Estimates for the errors in the Rayleigh-Ritz method...... 153

M. H. Martin, The Monge-Ampère partial differential equation

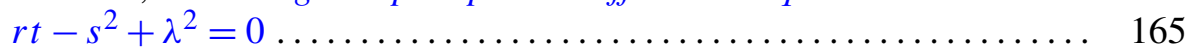

John E. Maxfield, Normal k-tuples ......................... 189

Jack E. McLaughlin, Structured theorems for relatively complemented lattices............................................ 197

William H. Mills, A system of quadratic Diophantine equations ......... 209

T. S. Motzkin, Ernst Gabor Straus and F. A. Valentine, The number of farthest points ................................. 221

G. Power, Forces on the boundary of a dielectric ............... 233

Ralph Gordon Selfridge, Approximations with least maximum error....... 247 